\title{
Percepción de enfermedad y características sociodemográficas en enfermos crónicos
}

\section{Disease perception and sociodemographic characteristics in chronic patients}

\author{
Japcy Margarita Quiceno ${ }^{1}$ y Stefano Vinaccia ${ }^{2}$
}

Citación: Quiceno, J.M. y Vinaccia, S. (2019). Percepción de enfermedad y características sociodemográficas en enfermos crónicos. Psicología y Salud, 29(2), 159-166.

RESUMEN

El propósito de este estudio fue evaluar las relaciones entre la percepción de enfermedad y las características sociodemográficas en enfermos crónicos con diagnóstico de insuficiencia renal crónica, artritis reumatoide y enfermedad pulmonar obstructiva crónica. La muestra estuvo conformada por 124 pacientes con una media de edad de 59 años. El diseño del estudio fue descriptivo correlacional y el instrumento utilizado fue el Cuestionario de Percepción de Enfermedad (IPQ-B). Las puntuaciones medias más altas en el IPQ-B se alcanzaron en las dimensiones Duración, Control personal, Control de tratamiento y Comprensión de la enfermedad, mientras que en las otras dimensiones hubo puntuaciones promedio. En cuanto a los factores que los pacientes consideraron que originaron su enfermedad, los porcentajes más altos de respuestas estuvieron relacionados con estilos de vida inadecuados y genética y herencia. En relación a las correlaciones entre el instrumento y las características sociodemográficas, se hallaron relaciones negativas entre la dimensión Consecuencias del IPQ-B con la edad y años cursados de estudios, y positivas entre la dimensión Comprensión de la enfermedad con los años cursados de estudio y el tiempo de diagnóstico. Se discuten los resultados con base en los datos encontrados.

Palabras clave: Percepción de enfermedad; Cuestionario de Percepción de Enfermedad; Enfermedad crónica; Factores sociodemográficos.

\begin{abstract}
The purpose of this study was to evaluate the relationship between the perception of disease and sociodemographic characteristics in chronic patients with a diagnosis of CRF, AR and COPD. The sample consisted of 124 patients with an average age of 59 years. The design of the study was descriptive-correlational, and the instrument used was the Brief Illness Perception Questionnaire. The highest average scores in the IPQ-B occurred in the dimensions: Duration, Personal control, Treatment control and Understanding the disease, with average scores in the other dimensions. Regarding the factors patients considered as origin of their disease, the highest percentages were related to inadequate lifestyle and genetic factors. Results between the IPQ-B and the sociodemographic characteristics, revealed negative correlations between the consequences dimension of the IPQ-B with age and schooling, and positive ones between the understanding dimension of IPQ-B disease with schooling and time since diagnosis.
\end{abstract}

Key words: Disease perception; Brief Illness Perception Questionnaire; Chronic disease; Sociodemographic factors.

\footnotetext{
${ }^{1}$ Universidad de Medellín, Carrera 87 N³0 - 65, Medellín, Colombia, tel. 57(4)340-55-55, correo electrónico: japcyps@hotmail.com. Artículo recibido el 22 de mayo y aceptado el 7 de agosto de 2018.

${ }^{2}$ Universidad del Sinú, Campus Elías Bechara Zainúm, Cra. 1w No. 38-153, Barrio Juan XxiII, Montería - Córdoba, Colombia, PBX (+1)781-17-17, correo electrónico: vinalpi47@hotmail.com.
} 


\section{INTRODUCCIÓN}

$\mathrm{L}$ a Organización Mundial de la Salud (OMS) (2018) ha definido la enfermedad crónica como aquel trastorno orgánico-funcional, que obliga a una modificación en el estilo de vida de un individuo y que es probable que persista por largo tiempo (Vinaccia, Quiceno y Medina, 2016). Las enfermedades crónicas son enfermedades multifactoriales, esto es, ocurren debido a factores genéticos, anatómicos, psicológicos y socioculturales; tienen un desarrollo progresivo, por lo que no es posible la recuperación completa; generalmente causan algún nivel de discapacidad permanente, y por lo regular no son infecciosas (Vinaccia y Orozco, 2005; Vinaccia y Quiceno, 2012).

La importancia relativa de las enfermedades crónicas en la sociedad ha aumentado significativamente en los últimos 70 años como resultado de los avances en la medicina, el desarrollo de la ingeniería sanitaria y la mejora sustancial en el estándar y la esperanza de vida, mismos que han reducido las enfermedades infecciosas. Pero ese interés también se debe a ciertos factores negativos asociados al estilo de vida, como los efectos del tabaquismo, el sedentarismo, el uso de alcohol, las dietas malsanas y la obesidad (Ward, Schiller y Goldman, 2014).

Las enfermedades crónicas son la causa de la muerte de 41 millones de personas anualmente ( $71 \%$ de todos los fallecimientos en el mundo). El mayor número de muertes se debe a las enfermedades cardiovasculares (17.9 millones), seguidas por tumores (9 millones), enfermedades respiratorias (3.9 millones) y diabetes (1.6 millones). Por otro lado, $13 \%$ de las muertes causadas por las enfermedades crónicas ocurre en los países desarrollados, registrándose el resto en países con niveles de ingreso medio-bajo, en los cuales vive la mayor parte de la población mundial (Global Burden of Disease [GBD], 2017). Aproximadamente entre 60 y $80 \%$ de todos los gastos de salud en todos los países se utilizan para el tratamiento de las enfermedades crónicas (Ogden, Carroll, Fryar y Flegal, 2015).

Por otro lado, independientemente de que sufran o no una enfermedad crónica, las personas tienen una concepción de salud y enfermedad que influye en el modo en que reaccionan ante los síntomas percibidos. Los llamados "esquemas de la enfermedad" son las representaciones organizadas de la misma que se adquieren a través de las experiencias de determinadas enfermedades que hayan padecido el mismo sujeto o sus familiares y amigos (Quiceno y Vinaccia, 2010).

Al respecto, Leventhal (1979) propuso un modelo jerárquicamente organizado con tres constructos principales: 1) "representación" de la experiencia de la enfermedad, 2) planificación de acción o respuestas de "afrontamiento" y 3) "evaluación" o monitoreo del éxito o fracaso de los esfuerzos de afrontamiento. Una característica novedosa de la proposición de Leventhal fue establecer dos canales paralelos para el sistema de procesamiento de la información: el primero para la representación objetiva de la enfermedad y el desarrollo de estrategias adecuadas de afrontamiento, y el segundo para el procesamiento de las respuestas emocionales producidas por la enfermedad y el desarrollo de estrategias de afrontamiento (cf. Cameron y Leventhal, 2003).

La construcción de este modelo de autorregulación, que también ha sido llamado del "sentido común", partió pues del postulado de que tales representaciones se integran con esquemas existentes, con las pautas normativas que las personas ya tenían previamente (Weinman, Petrie, Moss-Morris y Horne, 1996). Estos esquemas son, a saber: identidad, es decir, la etiqueta o nombre dado a la condición y a los síntomas que identifican una enfermedad; causa, que se refiere a las ideas que tiene el paciente sobre las causas de su enfermedad; tiempo, o sea, la creencia predictiva sobre cuánto tiempo puede durar la misma; consecuencias, o sea, las creencias individuales sobre las consecuencias de la condición y cómo estas tendrán un impacto físico y social, y curabilidad/controlabilidad, que se refiere a las creencias sobre si la condición puede curarse o mantenerse bajo control y el grado en que la persona desempeña un papel para lograrlo (Weinman y Petrie, 1997; Weinman, Petrie, Sharpe y Walker, 2000).

Además, es importante señalar que las creencias, las percepciones y las clasificaciones sociales e individuales sobre el proceso de salud-enfermedad pueden también estar determinadas por el contexto sociocultural y el momento histórico en 
que se manifiesten, incorporándose así a las creencias populares, que funcionan como esquemas cognitivos estereotipados que guían el comportamiento inmediato de las personas, permitiéndoles la rápida evaluación de una determinada enfermedad (Ehrenzweig, 2005). Por otro lado, el modelo de autorregulación de Leventhal para encarar la enfermedad habla de la importancia de las variables sociodemográficas en la susceptibilidad y en la gravedad percibidas (Marks et al., 2008).

Así, el objetivo del presente estudio fue evaluar las relaciones entre la percepción de enfermedad y algunas características sociodemográficas en enfermos crónicos colombianos de la ciudad de Medellín, departamento de Antioquia, con diagnóstico de insuficiencia renal crónica (IRC), artritis reumatoide (AR) y enfermedad pulmonar obstructiva crónica (EPOC).

\section{MÉTODO}

\section{Participantes}

En este estudio exploratorio, transversal, comparativo, ex post facto correlacional, hecho mediante una metodología tipo encuesta, según los criterios de Montero y León (2002), participaron 121 personas escogidas mediante un muestreo no aleatorio de sujetos disponibles, quienes fueron divididos en tres grupos según el tipo de enfermedad: 40 con IRC sometidos a hemodiálisis en el Instituto Clínica del Riñón de Medellín de Fresenius Medical Care de Colombia, diagnosticados según los criterios de Lameire, Van Biesen y Vanholder (2005); 41 con AR en tratamiento ambulatorio en la Fundación para el Paciente con Artritis Reumatoide (FUNPAR) de Medellín, diagnosticados según los criterios de Arnett et al. (1988), y 40 con EPOC, hospitalizados en el Hospital General de Medellín "Luz Castro de Gutiérrez", diagnosticados según los criterios de Dewar y Curry (2006).

\section{Instrumento}

Cuestionario de Percepción de Enfermedad (Brief Illness Perception Questionnaire [IPQ-B]). Desarrollado originalmente por Broadbent, Petrie, Main y Weinman (2006) y en versión española de Pacheco et al. (2012), este cuestionario está conformado por nueve ítems con respuestas tipo Likert que van de 0 a 10 puntos. Comprende ocho dimensiones y una pregunta abierta ordinal con tres posibilidades de respuesta. Las dimensiones son las siguientes: Consecuencias, Duración, Control personal, Control del tratamiento, Identidad, Preocupación, Respuesta emocional y Comprensión de la enfermedad. El ítem número 9 es una pregunta abierta que hace referencia a los factores que, según la persona, causaron su enfermedad, organizados según su importancia.

\section{Procedimiento}

Antes de iniciar la fase de campo, el estudio fue aprobado por el Comité de Ética Investigativa de las Universidades del Sinú y de Medellín. Después se llevó a cabo el proceso de adaptación cultural del IPQ-B, según los criterios de Alexandre y Guirardello (2002). Dicha adaptación, hecha sobre la base de la versión española de Pacheco et al. (2012), consistió en la aplicación de las pruebas a un grupo de 18 pacientes crónicos de ambos sexos con diagnóstico de diabetes tipo II. No se encontraron deficiencias en el instrumento que ameritaran cambios en su redacción, pues los pacientes reportaron haber comprendido a cabalidad los ítems en todos los instrumentos psicométricos evaluados; por consiguiente, previo permiso y autorización de las tres diferentes instituciones (una clínica renal, una fundación para pacientes con artritis reumatoide y un hospital público), se llevaron a cabo las aplicaciones del IPQ-B. Antes de las aplicaciones de los cuestionarios, a todos los participantes se les solicitó su consentimiento informado. Finalmente, se aplicaron los cuestionarios de una forma autoadministrada, interviniendo los investigadores solamente en el caso de que los pacientes requirieran algún tipo de ayuda. El tiempo de aplicación fue de 10 minutos en promedio.

\section{RESULTADOS}

\section{Descripción de la muestra según las características sociodemográficas}

La Tabla 1 muestra las características sociodemográficas y clínicas de los participantes en el estu- 
dio, todos ellos pacientes voluntarios de entre 22 y 86 años, de ambos sexos, con diagnóstico médico

de IRC, AR o EPOC. En general, hubo un predominio del género femenino.

Tabla 1. Características sociodemográficas y clínicas.

\begin{tabular}{|c|c|c|c|c|c|}
\hline Características & $\mathbf{n}$ & $\%$ & Características & $\mathbf{n}$ & $\%$ \\
\hline \multicolumn{3}{|l|}{ Sexo } & \multicolumn{3}{|l|}{ Escolaridad (años) } \\
\hline Mujeres & 76 & 62.8 & Media (D.T.) & \multicolumn{2}{|c|}{$7.34(5.0)$} \\
\hline Hombres & 45 & 37.2 & Rango (min.-max.) & \multicolumn{2}{|c|}{$(1-20)$} \\
\hline \multicolumn{3}{|l|}{ Edad (años) } & \multicolumn{3}{|l|}{ Ocupación } \\
\hline Media (D.T.) & \multicolumn{2}{|c|}{$59.18(13.3)$} & Empleado & 5 & 4.1 \\
\hline Rango (min.-max.) & \multicolumn{2}{|c|}{$(22-86)$} & Trabajador independiente & 13 & 10.7 \\
\hline \multicolumn{3}{|l|}{ Estado civil } & Jubilado/Pensionado & 39 & 32.2 \\
\hline Soltero & 28 & 23.1 & Desempleado & 12 & 9.9 \\
\hline Casado & 54 & 44.6 & Ama de casa & 52 & 43.0 \\
\hline Separado/Divorciado & 18 & 14.9 & \multicolumn{3}{|l|}{ Estrato socioeconómico } \\
\hline Unión Libre & 6 & 5.0 & Bajo (niveles 1 y 2 ) & 59 & 48.8 \\
\hline Viudo & 15 & 12.4 & Medio (niveles 3 y 4 ) & 55 & 45.5 \\
\hline \multicolumn{3}{|l|}{ Con quién vive } & Alto (niveles 5 y 6 ) & 7 & 5.8 \\
\hline Solo & 8 & 6.6 & \multicolumn{3}{|c|}{ Tiempo de diagnóstico (meses) } \\
\hline Con familia & 111 & 91.7 & Media (DT) & 85.56 & 85.6 \\
\hline Con amigos & 2 & 1.7 & Rango (Min-Max) & \multicolumn{2}{|c|}{$(1-444)$} \\
\hline \multicolumn{3}{|l|}{ Sistema de salud } & \multicolumn{3}{|c|}{ Consumo de medicación psiquiátrica } \\
\hline SISBEN & 39 & 32.2 & $\mathrm{Si}$ & 6 & 5.0 \\
\hline EPS & 79 & 65.3 & No & 115 & 95.0 \\
\hline Medicina prepagada & 2 & 1.7 & \multicolumn{3}{|c|}{ En tratamiento farmacológico } \\
\hline Particular/Privada & 1 & 0.8 & Sí & 117 & 96.7 \\
\hline & & & No & 4 & 3.3 \\
\hline
\end{tabular}

SISBEN $=$ Sistema de identificación de potenciales beneficiarios de programas sociales.

EPS $=$ Entidad prestadora de servicios.

En la Tabla 2 se muestran las diferencias entre las variables sociodemográficas y clínicas y las varia- bles psicológicas de la muestra total de enfermos con IRC, AR y EPOC).

Tabla 2. Análisis descriptivo del cuestionario IPQ-B $(\mathrm{n}=121)$.

\begin{tabular}{|l|c|c|c|}
\cline { 2 - 4 } \multicolumn{1}{l|}{} & Media & Mediana & D.T. \\
\hline Consecuencias & 6.4 & 7.0 & 3.5 \\
\hline Duración & 7.0 & 9.0 & 3.5 \\
\hline Control personal & 7.6 & 8.0 & 2.7 \\
\hline Control de tratamiento & 8.4 & 9.0 & 2.2 \\
\hline Identidad & 5.1 & 5.0 & 3.3 \\
\hline Preocupación & 5.5 & 6.0 & 3.6 \\
\hline Comprensión de la enfermedad & 7.8 & 9.0 & 2.8 \\
\hline Respuesta emocional & 4.8 & 5.0 & 3.6 \\
\hline
\end{tabular}

Factores que causaron la enfermedad: Ítem No. 9 del Cuestionario IPQ-B

Como ya se dijo antes, el IPQ-B incluye una novena pregunta abierta de tipo cualitativo en la que el paciente señala las causas que, en orden de impor- tancia, considera que causaron la enfermedad. Las respuestas dadas por los pacientes fueron clasificadas en nueve categorías. En la Tabla 3 aparecen las frecuencias por categoría de cada uno de los tres grupos del estudio. 
Tabla 3. Ítem 9 del IPQ-B: Factores que causaron la enfermedad según los pacientes.

\begin{tabular}{|c|c|c|c|c|c|c|c|c|c|c|}
\hline \multirow{2}{*}{ Respuestas } & \multicolumn{2}{|c|}{ IRC } & \multicolumn{2}{|c|}{ AR } & \multicolumn{2}{|c|}{ EPOC } & \multirow{2}{*}{$\mathbf{n}$} & \multirow{2}{*}{$\mathrm{X}^{2}$} & \multirow{2}{*}{ df } & \multirow{2}{*}{$p$} \\
\hline & Frec. & $\%$ & Frec. & $\%$ & Frec. & $\%$ & & & & \\
\hline No sabe/No responde & 6 & 15 & 4 & 10 & 4 & 10 & 14 & \multirow{7}{*}{74.237} & \multirow{7}{*}{16} & \multirow{7}{*}{0.000} \\
\hline Genética / Herencia & 6 & 15 & 19 & 46 & 2 & 5 & 27 & & & \\
\hline Enfermedad física & 18 & 45 & 3 & 7 & 2 & 5 & 23 & & & \\
\hline Estilos de vida inadecuados & 5 & 13 & 2 & 5 & 16 & 40 & 23 & & & \\
\hline Eventos vitales estresantes & 1 & 3 & 4 & 10 & 2 & 5 & 7 & & & \\
\hline Trabajo & 0 & 0 & 2 & 5 & 5 & 13 & 7 & & & \\
\hline Trascendental & 1 & 3 & 3 & 7 & 8 & 20 & 12 & & & \\
\hline
\end{tabular}

Se pueden apreciar en la tabla las diferentes respuestas que dieron los pacientes que participaron en el estudio sobre los tres factores más importantes que causaron su enfermedad en orden de importancia para cada uno de ellos.

En los pacientes con IRC se encuentra como primera causa de la enfermedad una enfermedad física, en los pacientes con AR, una causa genética o heredada, y en los pacientes con EPOC los estilos de vida inadecuados. Es de resaltar que solo los pacientes con EPOC consideraron que la enfermedad había sido causada por factores trascendentales.

Tabla 4. Correlación de Pearson entre percepción de enfermedad y edad, años de estudios y tiempo de diagnóstico.

\begin{tabular}{|l|c|c|c|}
\hline \multicolumn{1}{|c|}{ Dimensiones del IPQ-B } & Edad & $\begin{array}{c}\text { Años cursados } \\
\text { de estudios }\end{array}$ & $\begin{array}{c}\text { Tiempo de diagnóstico } \\
\text { de la enfermedad (meses) }\end{array}$ \\
\hline Consecuencias & $-0.245^{* *}$ & $-0.335^{* *}$ & -0.144 \\
\hline Duración & -0.012 & 0.011 & 0.057 \\
\hline Control personal & $-0.190 *$ & 0.162 & -0.046 \\
\hline Control de tratamiento & -0.082 & 0.120 & 25 \\
\hline Identidad & $-0.200 *$ & -0.045 & 0.112 \\
\hline Preocupación & 0.080 & $-0.262 * *$ & -0.141 \\
\hline Comprensión de enfermedad & -0.117 & $0.202 *$ & $0.258 * *$ \\
\hline Respuesta emocional & 0.079 & $-0.188^{*}$ & 0.154 \\
\hline$* p<.05$, dos colas. & & \\
$* * p<.01$, dos colas. & &
\end{tabular}

Se evidencian en la Tabla 4 las correlaciones moderadas positivas y negativas en los niveles de 0.01 y 0.05 . En general, se encontraron correlaciones negativas entre los años cursados de estudio con las dimensiones Consecuencias y Preocupación del IPQ-B, y positivas entre el tiempo de diagnóstico y la dimensión Comprensión de la enfermedad.

\section{DISCUSIÓN}

En cuanto a la percepción de la enfermedad medida con el cuestionario IPQ-B, se evidenció que los ítems relacionados con la sección de representación cognitiva de la enfermedad, como control personal, control del tratamiento, consecuencias y duración, obtuvieron los puntajes más altos, mientras los de la sección de representación emocional, como preocupación y respuesta emocional, los puntajes más bajos. Ello implica que los pacientes crónicos de esta muestra estaban convencidos e implicados en su tratamiento, lo que es alentador dada la evidencia de que las cogniciones relacionadas con el control pueden tener efectos protectores en los resultados relacionados con la salud. La dimensiones de control de la percepción de la enfermedad se refieren a la sensación de empoderamiento respecto a la efectividad de las conductas de afrontamiento (Hagger y Orbell, 2003), y es probable que influyan en la motivación del 
paciente para su cuidado personal. Por otro lado, los puntajes en las dimensiones Preocupación y Respuesta emocional muestran que, en términos generales, los pacientes no estaban demasiado preocupados y atentos a los signos y síntomas físicoemocionales de la enfermedad. La frecuencia de respuestas negativas a la percepción de la enfermedad es notable, pues se ha demostrado que influyen negativamente en el ajuste emocional y en los resultados relacionados con la salud en poblaciones con enfermedades crónicas (Cherrington, Moser, Lennie y Kennedy, 2004). Además, las muy razonables expectativas acerca de la evolución y el tiempo que durará la enfermedad son significativas en virtud de que la comprensión compartida de la enfermedad entre el médico y paciente es beneficiosa para facilitar la toma de decisiones, formular planes terapéuticos aceptables para este último y mejorar la adherencia al tratamiento (Street y Haidet, 2011).

Respecto al ítem 9 del cuestionario IPQ-B (“¿Cuáles son los factores más importantes que causaron su enfermedad?"), los pacientes crónicos fueron muy conscientes de las razones que los llevaron a enfermar y no distan mucho de lo reportado en la literatura científica. Entre las razones expuestas se hallan los estilos de vida inadecuados, la enfermedad física, la herencia y el trabajo; pero llama la atención el bajo porcentaje de factores trascendentales que consideraron los pacientes como causas de su enfermedad (la religión, la mala suerte o el destino), así como la ignorancia y la falta de respuesta, con excepción de $20 \%$ de pacientes con EPOC. Dichos pacientes eran los de mayor edad, con menos estudios y de un estrato socioeconómico más bajo. Esto último es significativo porque en estudios recientes, como el de Miglioretti, Meroni, Baiardo, Savioli y Velasco (2018), hecho en 211 pacientes cardiacos, empleando esa pregunta abierta del IPQ-B, 26\% no reconoció ninguna atribución causal. La probabilidad de que los pacientes no respondieran aumentó en los pacientes mayores, en las mujeres $\mathrm{y}$ en los pacientes con menores niveles educativos. Sobre eso mismo, Hoth, Wamboldt, Bowler, Make y Holm (2011), utilizando una muestra de 394 pacientes con EPOC, hallaron que 93\% de los mismos estuvieron de acuerdo o muy de acuerdo en que fumar era la causa de su condición. Es ne- cesario anotar que los sujetos del estudio anterior, quienes atribuyeron su enfermedad a algunos factores psicológicos, tenían más probabilidades de tener un reducido ajuste emocional y pobre calidad de vida.

Con relación a la búsqueda de relaciones entre la percepción de enfermedad (IPQ-B) y ciertas variables sociodemográficas (años cursados de estudio, edad y tiempo de diagnóstico de la enfermedad), en este último análisis se encontró que el número de años cursados de estudio se relacionaba negativamente con los sentimientos de preocupación y consecuencias (percepciones) acerca de las implicaciones físicas, sociales y económicas que tendrá la enfermedad, así como a las reacciones emocionales que podrán surgir como consecuencias de la misma. Adler y Ostrove (1999) y Lambregts (2017) plantean al respecto que los pacientes con antecedentes educativos más bajos tienen menos conocimientos sobre cómo reducir la posibilidad de recurrencia o tener un mejor control de los síntomas, lo que implicaría un aumento de emociones negativas. De igual manera, se halló que la edad de los pacientes también se relacionaba negativamente con la identificación de los síntomas físicos y emocionales y con la percepción de consecuencias severas de la enfermedad. Sobre esto mismo, Al-Smadi, Ashour, Hweidi, Gharaibeth y Fitzsimons (2016), en una revisión sistemática hecha con enfermos crónicos coronarios, encontraron que los pacientes mayores tenían una menor percepción de las consecuencias y de la cronicidad de su enfermedad. El número de meses desde el diagnóstico de la enfermedad se relacionó positivamente con los niveles de compresión de la misma.

Barraza y Sarmiento (2014) llevaron a cabo una investigación para evaluar el conocimiento de 150 pacientes hipertensos colombianos acerca de su enfermedad. Los resultados indican que quienes llevaban once años o más con la enfermedad tenían un conocimiento notablemente superior al de los recién diagnosticados.

Comprender la percepción que tienen los pacientes de la enfermedad que padecen podría permitir al paciente, a su familia y al médico adoptar medidas encaminadas a diseñar intervenciones más puntuales y eficaces para el manejo integral de aquélla aumentando la adherencia al tratamiento, 
disminuyendo la morbilidad asociada y, por supuesto, reduciendo los costos derivados de la atención (Valencia et al., 2014).

Debe considerarse que los grupos de pacientes con IRC y AR del presente estudio eran "profesionales de su enfermedad", por así decir. Aquellos con IRC estaban en un tratamiento altamente tecnificado de su enfermedad, con capacitación y apoyo psicológico en su centro de diálisis no solamente por parte de los profesionales sino por sus propios compañeros. Los pacientes con AR estaban asociados a un organismo no gubernamental que les brindaba apoyo educativo, legal y médico sobre su enfermedad, y los hacía participar en actividades recreativas de grupo y terapia ocupacional. Por el contrario, los pacientes con EPOC eran "novatos" respecto a su enfermedad, estaban aislados, y por su mayor edad y bajo nivel educativo tenían dificultades para entender el proceso patológico que los había llevado a hospitalizarse, visto más como gripe o influenza o como una enfermedad infecciosa aguda que como la enfermedad crónica que es.

La principal limitación de este estudio puede atribuirse al hecho de que los datos fueron recogidos en un momento específico de la enfermedad, cuando los pacientes se hallaban en un proceso de tratamiento y rehabilitación. Tales datos sugieren que la percepción del paciente acerca de su enfermedad se asocian moderadamente con variables tales como la edad, educación y tiempo de diagnóstico, factor este último que también fue encontrado en la investigación de Xavier et al. (2016) sobre la educación en pacientes de EPOC.

Por último, las investigaciones futuras sobre la influencia de los factores sociodemográficos sobre la percepción de la enfermedad deberían determinar si tales factores cambian o están relacionados con grupos específicos de pacientes que también comparten características específicas de cada enfermedad.

\section{REFERENCIAS}

Adler, N.E. y Ostrove, J.M. (1999). Socioeconomic status and health: What we know and what we don't. Annals of the New York Academy of Sciences, 896(1), 3-15.

Alexandre, N.M.C. y Guirardello, E.B. (2002). Adaptación cultural de instrumentos utilizados en salud ocupacional. Revista Panamericana de Salud Pública, 11(2), 109-111.

Al-Smadi, A.M., Ashour, A., Hweidi, I., Gharaibeth, B. y Fitzsimons, D. (2016). Illness perception in patients with coronary artery disease: A systematic review. International Nursering Practice, 22(6), 633-648.

Arnett, F.C., Edworthy, S.M., Bloch, D.A., McShane, D.J., Fries, J.F., Cooper, N.S. et al. (1988). The American Rheumatism Association 1987 revised criteria for the classification of rheumatoid arthritis. Arthritis Rheumatoid Review, 31 (3), $315-324$.

Barraza L., F. y Sarmiento C., A. (2014). Conocimiento de la enfermedad en una población hipertensa colombiana. Revista de la Facultad de Medicina, 62(3), 399-405.

Broadbent, E., Petrie, K.J., Main, J. y Weinman, J. (2006). The Brief Illness Perception Questionnaire. Journal of Psychosomatic Research, 60, 631-637.

Cameron, L. y Leventhal, H. (2003). The self-regulation of health and illness behaviour. New York: Psychology Press.

Cherrington, C.C., Moser, D.K., Lennie, T.A. y Kennedy, C.W. (2004). Illness representation after acute myocardial infarction: impact on in-hospital recovery. American Journal of Critical Care, 13(2), 136-145.

Dewar, M. y Curry, R.W. (2006). Chronic obstructive pulmonary disease: Diagnostic considerations. American Family Physician, 73, 669-678.

Ehrenzweig, Y. (2005). Modelos de cognición social y adherencia terapéutica en pacientes con cáncer. En L. Flores, M. M. Botero y B. Moreno (Eds.): Psicología de la salud: temas actuales de investigación en Latinoamérica (pp. 133-152). Barranquilla (Colombia): Uninorte.

Global Burden of Disease (2017). Global, regional, and national comparative risk assessment of 84 behavioral, environmental and occupational, and metabolic risks or clusters of risks, 1990-2016: a systematic analysis for the Global Burden of Disease Study 2016. The Lancet, 16(390), 1345-1422.

Hagger, M. y Orbell, S. (2003). A meta-analytic review of the common-sense model of illness representations. Psychology and Health, 18, 141-184.

Hoth, K., Wamboldt, F., Bowler, R., Make, B. y Holm, E. (2011). Attributions about cause of illness in chronic pulmonary disease. Journal of Psychosomatic Research, 70(5), 465-472. 
Lambregts, A. (2017). The relationship between illness perception and health-related quality of life among colorectal cancer patients: the role of socioeconomic status. Thesis submitted for the degree of B.Sc. Tilburg (Holanda): Tilburg University.

Lameire, N., Van Biesen, W. y Vanholder, R. (2005). Acute renal failure. Lancet, 365, 417-430.

Leventhal, H. (1979). A perceptual-motor processing model of emotion. En P. Pliner, K. Blankestein e I. M. Spiegel (Eds.): Perception of emotion in self and others (v. 5, pp. 1-46). New York: Plenum.

Marks, D., Murray, M., Evans, B., Willig, C., Woodall, C. y Sykes, C. (2008). Psicología de la salud: Teoría, investigación y práctica. México, D.F.: El Manual Moderno.

Miglioretti, M., Meroni, C., Baiardo, G., Savioli, G. y Velasco, V.(2018). The perceptions of the causes of cardiac disease: a taxonomy. Psychology \& Health, 33(4), 537-554.

Montero, I. y León, O. (2002). Clasificación y descripción de las metodologías de investigación en psicología. International Journal of Clinical and Health Psychology, 2, 503-508.

Ogden, C.L., Carroll, M.D., Fryar, C.D. y Flegal, K.M. (2015). Prevalence of obesity among adults and youth: United States, 2011-2014, NCHS Data Brief, 219, 1-8.

Organización Mundial de la Salud (2018). Enfermedades crónicas y promoción de la salud. Ginebra: OMS. Disponible en http:// www.who.int/chp/chronic_disease_report/part1/es/.

Pacheco H., V., Viladrich, C., Pujol R., E., Cabezas P., C., Núñez, M., Roura O. et al. (2012) Perception in chronic illnesses: linguistic validation of the revised Illness Perception Questionnaire and the Brief Illness Perception Questionnaire for a Spanish population. Atención Primaria, 44(5), 280-287.

Quiceno, J.M. y Vinaccia, S. (2010). Percepción de enfermedad: Una aproximación a partir del 'Illness Perception Questionnaire. Psicología desde el Caribe, 25, 56-83.

Street, R.L. y Haidet, P. (2011). How do doctors know their patients? Factors affecting physician understanding of patients health beliefs. Journal of General Internal Medicine, 26(1), 21-27.

Valencia, P., Mora, C., Arbelaez, A.M., Jaimes, Y., Merideidy, Y., Romero, M.C. et al. (2014). Cual es la percepción de enfermedad en pacientes colombianos con lupus eritematoso sistémico? Aplicación del Revised Percepción Questionnaire (IPQ-R). Revista Colombiana de Reumatología, 21, 4-9.

Vinaccia, S. y Orozco, L. (2005). Aspectos psicosociales asociados a la calidad de vida en pacientes crónicos. Diversitas: Perspectivas en Psicología, 1, 125-137.

Vinaccia, S. y Quiceno, J.M. (2012).Calidad de vida relacionada con la salud en enfermos crónicos colombianos. Revista Latinoamericana de Psiquiatría, 13(4), 156-163.

Vinaccia, S., Quiceno, J.M. y Medina A., M (2016). Conducta de enfermedad en pacientes con diagnóstico de dispepsia funcional y enfermedad pulmonar obstructiva crónica (EPOC): Un estudio transcultural. Psicología y Salud, 26(1), 5-14.

Ward, B.W. Schiller, J.S. y Goodman, R.A. (2014). Multiple chronic conditions among US adults: a 2012 update. Prevention of Chronic Disease, 2(11), E62.

Weinman, J. y Petrie, K.J. (1997). Illness perceptions: A new paradigm for psychosomatics? Journal of Psychosomatic Research, 42, 113-116.

Weinman, J., Petrie, K.J., Sharpe, N. y Walker, S. (2000). Causal attributions in patients and spouses following first-time myocardial infarction and subsequent lifestyle changes. British Journal of Health Psychology, 5, 263-273.

Weinman, J., Petrie, K., Moss-Morris, R. y Horne, R. (1996). The Illness Perception Questionnaire: A new measure for assessing the cognitive representation of illness. Psychology \& Health, 11, 431-445.

Xavier, A., Costa, A., Alves A., C., Carvalho P., R.M., Leon, F., Arrabal, S. et al. (2016). Association of illness perception with psycho-demographic factors and psysical activity in COPD patients. European Respiratory Journal, 8, PA 1890. doi: $10.1183 / 13993003$. 